Vol. $71(2005)$ [471-478]

\title{
CARTER SUBGROUPS IN THE GROUP OF UNITS OF AN ASSOCIATIVE ALGEBRA
}

\author{
Thorsten Bauer and Salvatore Siciliano
}

\begin{abstract}
In this paper we examine some properties of the Carter subgroups in the group of units of certain associative algebras. A description of the Carter subgroups in the case of a solvable associative algebra is obtained. Moreover, given an associative algebra $A$, we study relationships between the Cartan subalgebras of the Lie algebra associated with $A$ and the Carter subgroups of the group of units of $A$.
\end{abstract}

\section{INTRODUCTION}

A subgroup $H$ of a group $G$ is called a Carter subgroup if $H$ is nilpotent and selfnormalising in $G$. By a famous theorem due to Carter ([1]), a finite solvable group contains exactly one conjugacy class of Carter subgroups and it is conjectured by several authors that an arbitrary finite group contains at most one conjugacy class of such subgroups (see for example, $[2,3,11]$ ). In general, Carter's theorem does not hold for infinite groups. The infinite dihedral group is a simple example of a solvable infinite group containing Carter subgroups which are not conjugate.

Recall that a nilpotent and self-normalising subalgebra of a finite-dimensional Lie algebra $L$ is called a Cartan subalgebra. Analogies between properties of Carter subgroups and properties of Cartan subalgebras have already been considered in various places (see for instance $[6,10])$. Given a unitary associative algebra $A$ of finite dimension over a field, we can consider both the group of units $A^{\star}$ of $A$ and, on the other hand, the Lie algebra $A_{\text {Lie }}$ associated to $A$ by means of the Lie product $(x, y)=x y-y x$ for every $x, y \in A$. From results obtained in [9] (see Theorem 3 of the next section) we know, in particular, that the Cartan subalgebras of $A_{\text {Lie }}$ are associative subalgebras of $A$. Following [5], we say that $A$ is solvable if $A / \operatorname{Rad}(A)$ is commutative. We present two results which exhibit a close relationship beetwen the Cartan subalgebras of $A_{\mathrm{Lie}}$, the Carter subgroups of $A^{*}$, and the complements of $\operatorname{Rad}(A)$ in $A$ in the solvable case. Our first main result is a group-theoretic analogue of a result previously proved for the Cartan subalgebras of $A_{\text {Lie }}$ (see Theorem 3 of Section 2):

Received 10th January, 2005

The authors wish to express their gratitude to prof. Hartmut Laue for his precious help in the preparation of this paper.

Copyright Clearance Centre, Inc. Serial-fee code: 0004-9727/05 \$A2.00+0.00. 
THEOREM 1. Let $A$ be a finite dimensional solvable associative algebra over a field $F$ such that $A / \operatorname{Rad}(A)$ is separable. A subset $C$ of $A$ is a Carter subgroup of $A^{\star}$ if and only if there exists a complement $T$ of $\operatorname{Rad}(A)$ in $A$ such that $C=C_{A^{*}}\left(T^{\star}\right)$.

As a consequence, if $F \neq \mathbb{F}_{2}$, the Carter subgroups of $A^{\star}$ are exactly the groups of units of the Cartan subalgebras of $A_{\mathrm{Lie}}$. In Section 4, we apply Theorem 1 in order to prove the following:

THEOREM 2. Let $F$ be a field and $G$ a finite group such that $F G$ is solvable and $F G / \operatorname{Rad}(F G)$ is separable. Then every Carter subgroup of $G$ is contained in a Carter subgroup of $(F G)^{\star}$.

\section{Preliminaries and notations}

Given a positive integer $n$, the set of all positive integers $\leqslant n$ will be denoted by $\underline{n}$. Throughout this paper, the term "associative algebra" means an associative unitary algebra of finite dimension over a field. For a subset $S$ of an associative algebra $A$ over a field $F$, we denote by $\langle S\rangle_{F}$ the vector space spanned by $S$. Obviously, if $S$ is a subgroup of $A^{\star}$ then $\langle S\rangle_{F}$ coincides with the associative subalgebra generated by $S$ in $A$. In the next lemma, we collect some known facts about associative algebras which will be used in the sequel.

Lemma 1. Let $A$ be an associative algebra over a field $F$. If $H$ is a complement of $\operatorname{Rad}(A)$ in $A$, the following hold:

(1) If $J$ is an ideal of $A$ such that $J \subseteq \operatorname{Rad}(A)$ then $1+J$ is a nilpotent normal subgroup of $A^{*}$.

(2) $A^{\star}=H^{\star} \ltimes(1+\operatorname{Rad}(A))$.

(3) $\left\langle H^{\star}\right\rangle_{F}$ is a complement of $\operatorname{Rad}(A)$ in $\left\langle A^{\star}\right\rangle_{F}$.

(4) If $A$ is solvable and $B$ is a subalgebra of $A$ then $\operatorname{Rad}(B)=B \cap \operatorname{Rad}(A)$.

In particular, the group of units of a solvable associative algebra is always a solvable group.

As usual, for a finite dimensional vector space $V$ and a linear tranformation $f$ of $V$, we denote by $V_{0}(f)$ the null Fitting component of $V$ with respect to $f$. More generally, if $\mathcal{L}$ is any subset of End $V$, we write $V_{0}(\mathcal{L})$ for the null Fitting component of $V$ with respect to $\mathcal{L}$, that is,

$$
V_{0}(\mathcal{L})=\left\{v \in V \mid \forall f \in \mathcal{L} \exists n \in \mathbb{N} \quad v f^{n}=0\right\} .
$$

Now let $L$ be a finite dimensional Lie algebra. Then it is well known that a nilpotent subalgebra $H$ of $L$ is a Cartan subalgebra if and only if $H=L_{0}(\operatorname{ad} H)$ (see for example, [4, Proposition 1, Chapter III,1]). 
We say that an element $x$ of an associative algebra $A$ over a field $F$ is semisimple if the minimum polynomial of $x$ has no multiple roots in any extension field of $F$. A torus of $A$ is defined to be an Abelian subalgebra of $A$ consisting of semisimple elements. In [9], the following results about the Cartan subalgebras of $A_{\mathrm{Lie}}$ were obtained:

\section{THEOREM 3. Let $A$ be an associative algebra over a field $F$.}

(1) A subset $H$ of $A$ is a Cartan subalgebra of $A_{\mathrm{Lie}}$ if and only if there exists a maximal torus $T$ of $A$ such that $H=C_{A}(T)$.

(2) If $A$ is solvable such that $A / \operatorname{Rad}(A)$ is separable, the maximal tori of $A$ are exactly the complements of $\operatorname{Rad}(A)$ in $A$.

COROLlary 1. Let $A$ be a solvable associative algebra over a field $F$ such that $A / \operatorname{Rad}(A)$ is separable. $A$ subset $H$ of $A$ is a Cartan subalgebra of $A_{\text {Lie }}$ if and only if there exists a complement $T$ of $\operatorname{Rad}(A)$ in $A$ such that $H=C_{A}(T)$.

\section{Carter subgroups of Solvable associative algebras}

In order to prove Theorem 1, the following preliminary result is needed which may be of independent interest:

Lemma 2. Let $A$ be a solvable associative algebra over a field $F$ such that $A / \operatorname{Rad}(A)$ is separable. If $N$ is a nilpotent subgroup of $A^{\star}$, then the subalgebra $\langle N\rangle_{F}$ is Lie nilpotent.

Proof: Put $B:=\langle N\rangle_{F}$ and consider a complement $H$ of $\operatorname{Rad}(B)$ in $B$. The proof consists in showing that $B=C_{B}(H)$ : from this the claim follows by applying Corollary 1 to the algebra $B$.

Let $\bar{h} \in H$ and $g_{1}, g_{2}, \ldots, g_{r} \in N, \alpha_{1}, \alpha_{2}, \ldots, \alpha_{r} \in F$ such that $\bar{h}=\sum_{i=1}^{r} \alpha_{i} g_{i}$. Since $B^{\star}=H^{\star} \ltimes(1+\operatorname{Rad}(B))$, for all $i \in r$ there exist $h_{i} \in H^{\star}$ and $y_{i} \in \operatorname{Rad}(B)$ such that $g_{i}=h_{i}\left(1+y_{i}\right)$. Then

$$
\bar{h}=\sum_{i=1}^{r} \alpha_{i} h_{i}\left(1+y_{i}\right)=\sum_{i=1}^{r} \alpha_{i} h_{i}+\sum_{i=1}^{r} \alpha_{i} h_{i} y_{i}
$$

hence $\sum_{i=1}^{r} \alpha_{i} h_{i} y_{i} \in H \cap \operatorname{Rad}(B)=\{0\}$. Moreover, for all $i \in \underline{x}$ we have that $h_{i}=g_{i}\left(1+y_{i}\right)^{-1} \in N(1+\operatorname{Rad}(B))$. It follows that

$$
H=\left\langle H^{\star} \cap(N(1+\operatorname{Rad}(B)))\right\rangle_{F} .
$$

Now put

$$
J:=\left\{x \in B \mid \forall h \in H \exists n \in \mathbb{N} x(\operatorname{ad} h)^{n} \in \operatorname{Rad}(B)^{2}\right\} .
$$

As $B$ is solvable, $J$ is an ideal of $B$. By (1) of Lemma $1,1+(\operatorname{Rad}(B) \cap J)$ is a normal subgroup of $B^{\star}$. Again by the solvability of $B$ it is immediate that $N^{\prime} \leqslant 1+\operatorname{Rad}(B)$. We show that $N^{\prime}$ is contained in $1+(\operatorname{Rad}(B) \cap J)$. 
Let $z \in N^{\prime}$. Then $x:=z-1$ is an element of $\operatorname{Rad}(B)$ and, in particular, $z^{-1}=\sum_{i \in \mathbb{N}_{0}}(-1)^{i} x^{i}$. Let $\widehat{h} \in H^{\star} \cap(N(1+\operatorname{Rad}(B)))$ and $g \in N, y \in \operatorname{Rad}(B)$ such that $\widehat{h}=g(1+y)^{-1}$. Then

$$
[z, g]=\left(\sum_{i \in \mathrm{N}_{0}}(-1)^{i} x^{i}\right) g^{-1}(1+x) g \equiv 1+x^{g}-x \quad \bmod \operatorname{Rad}(B)^{2}
$$

Since $(1+y)^{-1}=\sum_{i \in N_{0}}(-1)^{i} y^{i}$, by (2) it follows that

$$
[z, g] \equiv 1+(1+y)^{-1} \widehat{h}^{-1} x \widehat{h}(1+y)-x \equiv 1+\widehat{h}^{-1}(x, \widehat{h}) \quad \bmod \operatorname{Rad}(B)^{2}
$$

From this, an easy induction shows that for every $n \in \mathbb{N}$

$$
\left[\left[\ldots[z, \underbrace{g] \ldots] g}_{n}] \in 1+\widehat{h}^{-n}\left(x(\operatorname{ad} \widehat{h})^{n}\right)+\operatorname{Rad}(B)^{2} .\right.\right.
$$

By the nilpotency of $N$, there exists a positive integer $m$ such that

$$
[[\ldots[z, \underbrace{g] \ldots] g}_{m}]=1 \text {. }
$$

By (3), this implies that $x(\operatorname{ad} \widehat{h})^{m} \in \operatorname{Rad}(B)^{2}$. Since the elements of

$$
H^{\star} \cap(N(1+\operatorname{Rad}(B)))
$$

commute mutually, by (1) it follows that $x \in J$. Hence

$$
N^{\prime} \subseteq 1+(\operatorname{Rad}(B) \cap J)
$$

as claimed above. We conclude that for every $a, a^{\prime} \in N$ there exists an element $s \in \operatorname{Rad}(B) \cap J$ such that $a a^{\prime}=a^{\prime} a(1+s)$, hence $\left(a, a^{\prime}\right)=a^{\prime} a s \in \operatorname{Rad}(B) \cap J$. In particular, $(B, H) \subseteq(B, B) \subseteq J$. It follows that for every $b \in B, h \in H$ there exists a positive integer $n$ such that $b(\operatorname{ad} h)^{n}$ is contained in $\operatorname{Rad}(B)^{2}$. This is the case $k=1$ of the following general assertion: For all $k \in \mathbb{N}, h \in H$ there exists a positive integer $n$ such that $\operatorname{Rad}(B)(\operatorname{ad} h)^{n} \subseteq \operatorname{Rad}(B)^{k+1}$. For a proof by induction on $k$, assume that for some positive integer $k$ there exists already a positive integer $m$ such that $\operatorname{Rad}(B)(\operatorname{ad} h)^{m}$ $\subseteq \operatorname{Rad}(B)^{k+1}$. Let $b \in \operatorname{Rad}(B), b^{\prime} \in \operatorname{Rad}(B)^{k}$. Then

$$
\left(b b^{\prime}\right)(\operatorname{ad} h)^{2 m}=\sum_{i=0}^{2 m}\left(\begin{array}{c}
2 m \\
i
\end{array}\right) b(\operatorname{ad} h)^{i} b^{\prime}(\operatorname{ad} h)^{2 m-i} \in(\operatorname{Rad}(B))^{k+2} .
$$

As $\operatorname{Rad}(B)^{k+1}$ is the linear span of finitely many of the elements $b b^{\prime}$, there exists a positive integer $l$ such that $\operatorname{Rad}(B)^{k+1}(\operatorname{ad} h)^{l} \subseteq \operatorname{Rad}(B)^{k+2}$. Now it suffices to put $n:=m+l$ to 
complete the inductive step. By the nilpotency of $\operatorname{Rad}(B)$, it follows that $B$ is contained in the null Fitting component $B_{0}(\operatorname{ad} h)$ for every $h \in H$. This means that $B \subseteq B_{0}(\operatorname{ad} H)$. Finally, as $H$ is a torus it follows that $B_{0}(\operatorname{ad} H)=C_{B}(H)$ (see [9]), and the proof is complete.

As usual, for a group $G$ we denote by $\gamma_{k}(G)$ the $k$-th term of the lower central series of $G$. We now prove our main result of this section.

Proof of Theorem 1: First assume that $T$ is a complement of $\operatorname{Rad}(A)$ in $A$. Since $A^{\star}=T^{\star} \ltimes(1+\operatorname{Rad}(A))$, for every $k \geqslant 2$ we have $\gamma_{k}\left(C_{A^{*}}\left(T^{\star}\right)\right) \subseteq \gamma_{k}\left(C_{1+\operatorname{Rad}(A)}\left(T^{\star}\right)\right)$. By (1) of Lemma $1,1+\operatorname{Rad}(A)$ is nilpotent, hence $C_{A^{*}}\left(T^{*}\right)$ is also nilpotent. Let $g \in N_{A^{*}}\left(C_{A^{*}}\left(T^{\star}\right)\right)$ and put $B:=\left\langle C_{A^{*}}\left(T^{\star}\right)\right\rangle_{F}$. By (3) of Lemma $1,\left\langle T^{\star}\right\rangle_{F}$ is a complement of $\operatorname{Rad}(B)$ in $B$. We have $T^{\star g} \subseteq C_{A^{*}}\left(T^{\star}\right)$, so that $\left\langle T^{\star}\right\rangle_{F}^{g}$ is a complement of $\operatorname{Rad}(B)$ in $B$, too. By the Wedderburn-Malcev Theorem, $T^{\star}$ and $T^{\star g}$ are conjugate under $\operatorname{Rad}(B)$. But $\left\langle T^{\star}\right\rangle_{F} \subseteq Z(B)$ now forces $\left\langle T^{\star}\right\rangle_{F}=\left\langle T^{\star}\right\rangle_{F}^{g}$, hence $T^{\star g}=T^{\star}$. It follows that

$$
\left[g, T^{\star}\right] \subseteq(1+\operatorname{Rad}(A)) \cap T^{\star}=\{1\},
$$

hence $g \in C_{A^{*}}\left(T^{\star}\right)$. Therefore $C_{A^{\star}}\left(T^{\star}\right)$ is a Carter subgroup of $A^{\star}$.

Conversely, let $C$ be a Carter subgroup of $A^{\star}$. Put $B:=\langle C\rangle_{F}$. By Lemma $2, B$ is Lie nilpotent. Theorem 3 implies that $B=C_{B}\left(T_{0}\right)$ for a complement $T_{0}$ of $\operatorname{Rad}(B)$ in $B$. As $B=\langle C\rangle_{F} \subseteq\left\langle B^{\star}\right\rangle_{F},(3)$ of Lemma 1 implies that $T_{0}=\left\langle T_{0}^{\star}\right\rangle_{F}$. Consequently, $B^{\star}=\left(C_{B}\left(T_{0}\right)\right)^{\star}=C_{B^{\star}}\left(T_{0}^{\star}\right)$ which, by the first part of the proof, is a Carter subgroup of $B^{\star}$. Hence $B^{\star}$ is nilpotent. Being a Carter subgroup, $C$ is in particular a maximal nilpotent subgroup of $B^{\star}$. It follows that $B^{\star}=C$. By (4) of Lemma 1 we have $\operatorname{Rad}(B+\operatorname{Rad}(A))=\operatorname{Rad}(A) . \quad T$ herefore $T_{0}$ is a complement of $\operatorname{Rad}(B+\operatorname{Rad}(A))$ in $B+\operatorname{Rad}(A)$. By the first part of the proof, $C_{(B+\operatorname{Rad}(A))^{*}}\left(T_{0}^{\star}\right)$ is a Carter subgroup of the $\operatorname{group}(B+\operatorname{Rad}(A))^{\star}=T_{0}^{\star} \ltimes(1+\operatorname{Rad}(A))$. As $C \leqslant C_{(B+\operatorname{Rad}(A))^{*}}\left(T_{0}^{\star}\right)$, it follows that $C=C_{(B+\operatorname{Rad}(A))^{*}}\left(T_{0}^{\star}\right)$. This yields

$$
\left[C_{A^{*}}\left(T_{0}^{\star}\right), C\right] \leqslant C_{A^{*}}\left(T_{0}^{\star}\right) \cap(1+\operatorname{Rad}(A)) \leqslant C
$$

and therefore $C_{A^{*}}\left(T_{0}^{\star}\right) \subseteq N_{A^{*}}(C)=C$. Now, by (2) of Theorem $3, T_{0}$ is a torus of $B$. Let $T$ be a maximal torus of $A$ which contains $T_{0}$. Then (2) of Theorem 3 implies that $T$ is a complement of $\operatorname{Rad}(A)$ in $A$. Again by the first part of the proof, $C_{A^{*}}\left(T^{\star}\right)$ is a Carter subgroup of $A^{\star}$ and we have $C_{A^{*}}\left(T^{\star}\right) \leqslant C_{A^{*}}\left(T_{0}^{\star}\right) \leqslant C$. Finally, as $C_{A^{*}}\left(T^{\star}\right)$ is a maximal nilpotent subgroup of $C$, it follows that $C=C_{A^{*}}\left(T^{\star}\right)$, completing the proof.

We have already remarked that the Carter subgroups of a solvable infinite group are not necessarily conjugate. Nevertheless, like in Carter's theorem, for the group of units of any solvable associative algebra we have:

COROLlary 2. Let $A$ be a solvable associative algebra over a field $F$ such that $A / \operatorname{Rad}(A)$ is separable. Then $A^{\star}$ contains exactly one conjugacy class of Carter subgroups. 
Proof: Theorem 1 and the Wedderburn-Malcev Theorem imply the existence of a Carter subgroup of $A^{\star}$. Let $C_{1}$ and $C_{2}$ be Carter subgroups of $A^{\star}$. Again by the quoted theorems, there exist complements $T_{1}$ and $T_{2}$ of $\operatorname{Rad}(A)$ in $A$ such that $C_{i}=C_{A^{*}}\left(T_{i}^{*}\right)$, and an element $x \in \operatorname{Rad}(A)$ such that $T_{2}=T_{1}^{1+x}$. Hence $C_{2}=C_{1}^{1+x}$, as required.

REMARK 1. Let $A$ be an associative algebra over a field $F$. Suppose that $A=F_{1} \oplus F_{2} \oplus \cdots \oplus F_{n}$, where each $F_{i}$ is an extension field of $F$ and $F_{i} \neq \mathbb{F}_{2}$. Then it is easy to see that $A$ is generated by $A^{\star}$ as a vector space.

COROLlary 3. Let $A$ be a solvable associative algebra over a field $F, F \neq \mathbb{F}_{2}$, such that $A / \operatorname{Rad}(A)$ is separable. Then the Carter subgroups of $A^{\star}$ are exactly the groups of units of the Cartan subalgebras of $A_{\text {Lie }}$.

Proof: Let $T$ be a complement of $\operatorname{Rad}(A)$ in $A$. By Remark 1 it follows that $T=\left\langle T^{\star}\right\rangle_{F}$. Consequently, we have $\left(C_{A}(T)\right)^{\star}=\left(C_{A}\left(T^{\star}\right)\right)^{\star}=C_{A^{\star}}\left(T^{\star}\right)$ so that, by Corollary 1 and Theorem 1 , the claim follows at once.

REMARK 2. In general, the previous result fails if $F=\mathbb{F}_{2}$. For example, consider the associative algebra $A$ consisting of all upper triangular $2 \times 2$ matrices over $\mathbb{F}_{2}$. Then the set $\mathcal{D}$ of all diagonal matrices is a Cartan subalgebra of $A_{\text {Lie }}$ but, clearly, $\mathcal{D}^{\star}=\{1\}$ is not a Carter subgroup of $A^{\star}$.

\section{Carter subgroups of Solvable group algebras}

Let $G$ be a finite group and $F$ a field. When are the Carter subgroups of $G$ contained in Carter subgroups of $(F G)^{\star}$ ? In this section, we give a positive answer to the question when $F G$ is solvable. Recall that the group algebra $F G$ is solvable if and only if $G$ is Abelian or char $F=p>0$ and the derived group of $G$ is a $p$-group.

We preliminarily show the following finite analogue of Theorem 1:

LemMa 3. Let $G$ be a finite group. Suppose that $G$ has a normal Sylow subgroup $N$ such that $G / N$ is Abelian. Then $C \subseteq G$ is a Carter subgroup of $G$ if and only if there exists a complement $H$ of $N$ in $G$ such that $C=C_{G}(H)$.

Proof: Note that $G$ is solvable of nilpotency length at most 2, therefore the Carter subgroups of $G$ coincide with the Sylow normalisers of $G$ (see $[8,9.5 .10]$ ). Let $H$ be a complement of $N$ in $G$. Then $G=H \ltimes N$ and $H$ is clearly an Abelian subgroup of $G$. Let $p_{1}, p_{2}, \ldots, p_{r}$ be the distinct prime divisors of $|H|$ and for each $i \in \underline{r}$ denote by $P_{i}$ the unique $p_{i}$-Sylow subgroup of $H$. Then $\left\{P_{1}, \ldots, P_{r}, P_{r+1}=N\right\}$ is clearly a Sylow basis for $G$. Let $C$ be the normaliser of this Sylow basis. Then

$$
C=\bigcap_{i=1}^{r+1} N_{G}\left(P_{i}\right)=\bigcap_{i=1}^{r} N_{G}\left(P_{i}\right)
$$


Let $i \in \underline{r}$ and $g \in N_{G}\left(P_{i}\right)$. Then $\left[g, P_{i}\right] \leqslant P_{i} \cap N=\{1\}$, hence $g \in C_{G}\left(P_{i}\right)$. Since $H=P_{1} P_{2} \ldots P_{r}$, it follows that

$$
C=\bigcap_{i=1}^{r} C_{G}\left(P_{i}\right)=C_{G}(H)
$$

and the first part of the proof is complete.

Conversely, assume that $C$ is a Carter subgroup of $G$. By the Schur-Zassenhaus Theorem (see $[8,9.1 .2]$ ) there exists a complement $H$ of $N$ in $G$. Let $\widetilde{C}:=C_{G}(H)$. By the first part of the proof, $\tilde{C}$ is a Carter subgroup of $G$. Consequently, Carter's theorem implies that $\widetilde{C}^{x}=C$ for some $x \in G$. It follows that $C=\left(C_{G}(H)\right)^{x}=C_{G}\left(H^{x}\right)$ which implies the claim.

Proof of Theorem 2: The claim is trivial when $G$ is Abelian. Assume $G$ is not Abelian and $F$ of characteristic $p>0$. Let $C$ be a Carter subgroup of $G$. As $F G$ is solvable, the derived group $G^{\prime}$ is a $p$-group. Hence $G$ has a normal Sylow $p$-subgroup $P$ and $G / P$ is Abelian. By Lemma 3 there exists a complement $H$ of $P$ in $G$ such that $C=C_{G}(H)$. Let $g \in H$ and put $n:=|H|$. Since $g^{n}-1=0$, it follows that the minimum polynomial $f_{g}$ of $g$ divides the polynomial $x^{n}-1$. As $p$ does not divide $n$, this implies that $f_{g}$ has no multiple root in any extension field of $F$. Thus, $H$ consists of semisimple elements of $F G$ which commute pairwise. By [9], it follows that $T:=\langle H\rangle_{F}$ is a torus of $F G$. Since 0 is the only element of $F G$ which is both nilpotent and semisimple, we have $T \cap \operatorname{Rad}(F G)=\{0\}$. By Wallace's theorem (see $[7,4.7]$ ),

$$
\operatorname{Rad}(F G)=\sum_{x \in P}(x-1) F G .
$$

In particular, $P$ is contained in $1+\operatorname{Rad}(F G)$. It follows that

$$
F G=\langle H \ltimes P\rangle_{F}=\langle H(1+\operatorname{Rad}(F G))\rangle_{F}=T+\operatorname{Rad}(F G) .
$$

Thus $T$ is a complement of $\operatorname{Rad}(F G)$ in $F G$. Put $\widetilde{C}=C_{(F G)^{*}}\left(T^{\star}\right)$. By Theorem $1, \widetilde{C}$ is a Carter subgroup of $(F G)^{\star}$. Now

$$
C=C_{G}(H) \subseteq\left(C_{F G}(H)\right)^{\star} \subseteq\left(C_{F G}\left(T^{\star}\right)\right)^{\star}=C_{(F G)^{*}}\left(T^{\star}\right)=\tilde{C}
$$

and the claim is proved.

In [9] it is proved that the Cartan subalgebras of the Lie algebra associated with a central division algebra are Abelian. As a contrast to the close relationship between Carter subgroups and Cartan subalgebras exhibited in the foregoing sections for the solvable case, we finally observe:

REMARK 3. Let $D$ be a central division algebra of dimension 4 over a field $F$ of characteristic $\neq 2$. Then $D^{\star}$ does not have an Abelian Carter subgroup. 
For a proof by contradiction, assume that there is an Abelian Carter subgroup $C$ of $D^{\star}$. Then $C$ contains an element $y \in D^{\star} \backslash F$. The subalgebra $F[y]$ of $D$ generated by $F$ and $y$ is a field of dimension 2 over $F$. We conclude that

$$
C \subseteq C_{D^{*}}(y)=C_{D^{*}}(F[y])=(F[y])^{\star} \subseteq N_{D^{*}}(C)=C .
$$

Hence $C=(F[y])^{\star}$ which yields $N_{D^{*}}(F[y])=(F[y])^{\star}$. On the other hand, as the characteristic of $F$ is odd, $F[y]$ is a Galois extension of $F$, hence there exists an automorphism of order 2 of $F[y]$ which fixes the elements of $F$. By the Noether-Skolem Theorem (see [7, 12.6]), this automorphism is induced by a conjugation by an appropriate element $x \in D^{\star}$. It follows that $x \in N_{D^{\star}}(F[y]) \backslash(F[y])^{\star}$, a contradiction.

\section{REFERENCES}

[1] R. Carter, 'On a class of finite soluble groups', Proc. London Math. Soc. 9 (1959), 623-640.

[2] F. Dalla Volta, A. Lucchini and M.C. Tamburini, 'On the conjugacy problem for Carter subgroups', Comm. Algebra 26 (1998), 395-401.

[3] L. Di Martino, M.C. Tamburini and A.E. Zalesskiř, 'Carter subgroups in classical groups', J. London Math. Soc. 55 (1997), 264-276.

[4] N. Jacobson, Lie algebras (Wiley Interscience, New York, 1962).

[5] S.A. Jennings, 'Central chains of ideals in an associative ring', Duke Math. J. 9 (1942), 341-355.

[6] C.H. Hallahan and J. Overbeck, 'Cartan subalgebras of meta-nilpotent Lie algebras', Math. Z. 116 (1970), 215-217.

[7] R.S. Pierce, Associative algebras (Springer-Verlag, New York, 1982).

[8] D.J.S. Robinson, $A$ course in the theory of groups (Springer-Verlag, New York, 1982).

[8] S. Siciliano, 'Cartan subalgebras in Lie algebras of associative algebras', (Preprint of the University of Lecce).

[10] E. Stitzinger, 'Theorems on Cartan subalgebras like some on Carter subgroups', Trans. Amer. Math. Soc. 159 (1971), 307-315.

[11] M.C. Tamburini and E.P. Vdovin, 'Carter subgroups in finite groups', J. Algebra 255 (2002), 148-163.

Schuetzenwall 43

D-24114 Kiel

Germany

e-mail: thorsten.bauer@ppi.de
Dipartimento di Matematica "E. De Giorgi"

Università degli Studi di Lecce

Via Provinciale Lecce-Arnesano

73100-Lecce

Italy

e-mail: salvatore.siciliano@unile.it 